\title{
Nordihydroguaiaretic acid inhibition of NFATc1 suppresses osteoclastogenesis and arthritis bone destruction in rats
}

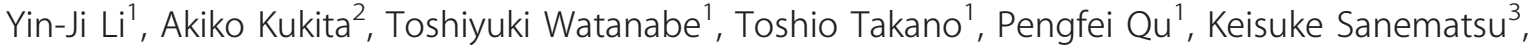 \\ Yuzo Ninomiya ${ }^{3}$ and Toshio Kukita ${ }^{1}$
}

Nordihydroguaiaretic acid (NDGA) is known to have prominent anticancer activity against several cancers, and is also known to be an inhibitor of 5-lipoxygenase (5-LO). In this study, we investigated the regulatory function of NDGA on inflammatory bone destruction mediated by osteoclasts. NDGA markedly inhibited receptor activator of nuclear factor- $\kappa \mathrm{B}$ (NF- $\kappa$ B) ligand (RANKL)-induced formation of osteoclasts in cultures of murine osteoclast precursor cell line RAW-D cells and primary bone marrow-derived macrophages culture systems. The inhibitory effect of NDGA on osteoclastogenesis did not arise from the inhibition of 5-LO activity. NDGA did not affect MAPKs, such as p38, JNK, and NF- $\kappa$ B, but significantly inhibited the induction of NFATc1, a key transcription factor for osteoclastogenesis. NDGA also suppressed activation of ERK in osteoclast precursors. RANKL-induced calcium oscillation observed in osteoclast precursors was completely diminished by the addition of NDGA. In mature osteoclasts, RANKL-induced nuclear translocation of NFATc1 was clearly inhibited by NDGA treatment. Finally, in vivo studies demonstrated that administration of NDGA significantly reduced severe bone destruction and osteoclast recruitment in the ankle joint of rats with adjuvant-induced arthritis. These results indicate the potential utility of NDGA as a therapeutic agent for ameliorating inflammatory bone destruction in rheumatoid arthritis.

Laboratory Investigation (2012) 92, 1777-1787; doi:10.1038/labinvest.2012.134; published online 8 October 2012

KEYWORDS: adjuvant arthritis; bone destruction; calcium oscillation; NDGA; NFATc1; RANKL

Rheumatoid arthritis (RA) is a systemic, chronic inflammatory autoimmune disease, accompanied by severe bone destruction. ${ }^{1,2}$ Bone destruction is mediated primarily by the bone-resorbing cells, osteoclasts, highly specialized multinucleated giant cells derived from hematopoietic stem cells. Abundant recruitment of osteoclasts is observed at sites of severe bone destruction in RA patients ${ }^{3}$ and in animal models of RA. ${ }^{4-6}$ Receptor activator of nuclear factor- $\kappa \mathrm{B}$ $(\mathrm{NF}-\kappa \mathrm{B})$ ligand (RANKL) is known as a key regulator of osteoclast differentiation and function in normal bone metabolism. ${ }^{7}$ RANKL binds to its receptor RANK and induces signaling events involving activation of MAPKs, calcium oscillation, and NFATc1. ${ }^{8}$ RANKL is also highly expressed in synovial fibroblasts and $\mathrm{T}$ cells in inflammatory synovial tissues, and is considered to have an important role in the aberrant stimulation of osteoclastogenesis in inflammatory lesions. ${ }^{4,9}$ Treatment with osteoprotegerin, the soluble decoy receptor for RANKL, ${ }^{10}$ has been shown to reduce osteoclast number and prevent bone erosion in collagen-induced arthritis. ${ }^{11}$ Bisphosphonates, pyrophosphate derivatives comprising a class of synthetic pharmacological agents used for blocking osteoclastogenesis, protect against bone loss in animal models of arthritis. ${ }^{12-14}$ Moreover, in the osteoclastfree animal model of arthritis, the decisive role of osteoclasts for arthritic bone erosion has been documented. ${ }^{15,16}$ Thus, controlling osteoclastogenesis is considered to be one effective approach for protection of bone destruction in RA. Recently, it has been noticed that administration of bisphosphonates induces bisphosphonate-related osteonecrosis of the jaw. Biological medicines, eg, anti-TNF antibody

\footnotetext{
${ }^{1}$ Faculty of Dental Science,Division of Oral Biological Sciences, Department of Molecular Cell Biology and Oral Anatomy, Kyushu University, Fukuoka, Japan; ${ }^{2}$ Faculty of Medicine, Department of Microbiology, Saga University, Saga, Japan and ${ }^{3}$ Faculty of Dental Science, Section of Oral Neuroscience, Kyushu University, Fukuoka, Japan Correspondence: Dr T Kukita, PhD, Faculty of Dental Science, Division of Oral Biological Sciences, Department of Molecular Cell Biology and Oral Anatomy, Kyushu University, 3-1-1 Maidashi, Fukuoka 812-8582, Japan, E-mail: kukitat@dent.kyushu-u.ac.jp or Dr A Kukita, PhD, Faculty of Medicine, Department of Microbiology, Saga University, 5-1-1 Nabeshima, Saga 849-8501, Japan, E-mail: kukita@cc.saga-u.ac.jp 
and anti-RANKL antibody, are now recognized as a powerful medicine to treat RA; however, these medicines could cause some side effects involving incidence of pneumonia.

Nordihydroguaiaretic acid (NDGA) is a phenolic compound originally isolated from the creosote bush (Larrea tridentata), which has been utilized from ancient days by native North Americans to treat various diseases, including arthritis and rheumatism. ${ }^{17}$ Recently, it has been revealed that NDGA possesses a potent anticancer activity against various cancer cells. NDGA inhibits proliferation of breast cancer cells through suppressing IGF-I receptor signaling. ${ }^{18}$ NDGA also inhibits growth of prostatic cancer cells through suppressing activation of IGF-I receptor. ${ }^{19}$ Intensive clinical trials are now in progress to develop NDGA as a potent anticancer drug for prostatic cancer. Thus, NDGA is supposed to have minimum side effects and could be developed as an effective regulator of bone destruction. NDGA is also known as an inhibitor of 5-lipoxygenase (5-LO), one of the key enzymes of the arachidonic acid pathways. ${ }^{17}$

In this study, we investigated the possible regulatory function of NDGA on osteoclastogenesis and found that NDGA significantly inhibited osteoclastogenesis in vitro. We also addressed the molecular mechanism of the inhibitory action of NDGA on osteoclastogenesis and obtained lines of evidence, suggesting that NDGA suppresses osteoclastogenesis through inhibiting NFATc1. In addition, we investigated the regulatory function of NDGA on inflammatory bone destruction in vivo in rats with adjuvant-induced arthritis (AA), accompanied by severe bone destruction around the ankle joints, and found that NDGA markedly inhibits bone destruction in AA rats.

\section{MATERIALS AND METHODS}

\section{Differentiation of Osteoclasts}

Osteoclast differentiation was induced from bone marrowderived macrophage (BMM) preparations. Briefly, bone marrow cells were isolated from the tibia and femur of 5-week-old male C57BL/6J mice. The bone marrow cells (2 to $3 \times 10^{7}$ cells in a $10-\mathrm{cm}$ dish) were cultured with $\alpha$-MEM (GIBCO, Grand Island, NY, USA) containing 10\% FBS (Biosource, Rockville, MD, USA) in the presence of $10 \mathrm{ng} / \mathrm{ml}$ M-CSF (PeproTech, London, UK) for $24 \mathrm{~h}$. The non-adherent cells were collected and cultured in $\alpha$-MEM containing $10 \%$ FBS and $20 \mathrm{ng} / \mathrm{ml} \mathrm{M}$-CSF. After 3 days, adherent cells were considered BMMs. For osteoclast generation, BMMs were further cultured in the presence of $50 \mathrm{ng} / \mathrm{ml}$ RANKL and $20 \mathrm{ng} / \mathrm{ml} \mathrm{M-CSF}$ for 5 days. To evaluate the effect of NDGA (Biomol International, Enzo Life Science, Farmingdale, NY, USA) on osteoclastogenesis, various concentrations of NDGA were added to these cultures at the same time as RANKL treatment. At the end of culture, the cells were fixed and stained for TRAP. TRAP-positive $(\mathrm{TRAP}+)$ multinucleated cells (MNCs) containing three or more nuclei were then counted. Osteoclast-like cells were also generated from
RAW-D cells, a subclone of the murine macrophage cell line, RAW264, having extremely high potential ability to differentiate into osteoclast-like MNCs. ${ }^{20}$ RAW-D cells were cultured in 96-well plates $\left(6.8 \times 10^{3}\right.$ cells per well $)$ in $\alpha$-MEM containing $10 \%$ FBS for 3 days in the presence of RANKL $(20 \mathrm{ng} / \mathrm{ml}), \quad$ TNF- $\alpha \quad(1 \mathrm{ng} / \mathrm{ml})$, and various concentrations of NDGA. After 3 days, the cells were stained for TRAP, and TRAP + MNCs were counted.

Osteoclasts were formed in rat whole bone marrow cell culture system as described previously. ${ }^{21,22}$ Briefly, bone marrow cells were obtained from the tibia and femur of 4-week-old male SD rats and cultured in 24-well plates $\left(1 \times 10^{6}\right.$ cells per well) in $\alpha$-MEM containing 15\% FBS in the presence of $10^{-8} \mathrm{M} 1 \alpha, 25(\mathrm{OH})_{2} \mathrm{D}_{3}$ and $10 \%$ heat-treated conditioned medium of osteoblast cell line, ROS 17/2.8. ${ }^{22}$ To confirm the formation of rat osteoclasts, cells were stained for osteoclast-specific antigen Kat1 as described previously. ${ }^{23-25}$

\section{Bone Resorption Assay}

Osteoclasts were formed in rat whole bone marrow cell culture system as described above. After 4 days of culture, the cells were detached from the culture plates with $0.05 \%$ trypsin and $0.02 \%$ EDTA. They were replated on a calciumphosphate thin layer-coated BioCoat Osteologic slides (BD Biosciences, Bedford, MA, USA) with $\alpha$-MEM containing $15 \%$ FBS in the presence or absence of NDGA. After 2 days of culture, attached cells were removed from the slides using 6\% sodium hypochlorite. Von Kossa staining was performed to stain unresorbed area, according to the manufacturer's protocol. Images of pits, identified as the unstained area, per well were obtained using inverted microscopy (BZ-8100; Keyence, Japan), and the total areas of resorption pits and the total number of resorption pits per well were determined using BZ analyzer software (Keyence).

\section{Cell Viability}

Cell viability was measured by MTT assay. Osteoclast-like MNCs from RAW-D cells or mouse BMMs were incubated with MTT for $4 \mathrm{~h}$ and lysed in lysing solution (20\% sodium dodecyl sulfate in $50 \%$ dimethylformamide). After overnight incubation, absorbance at $595 \mathrm{~nm}$ was determined with a microplate reader.

\section{Reverse Transcription PCR}

RAW-D cells were cultured in six-well plates $\left(1.5 \times 10^{5}\right.$ cells per well) with RANKL and TNF- $\alpha$ for the indicated times. Total cellular RNA was extracted using TRIzol Reagent (Invitrogen, Carlsbad, CA, USA) and subjected to reverse transcription PCR (RT-PCR) using a RT-PCR kit (Takara Bio, Otsu, Japan). The primers used for PCR were as follows: ATP6V0d2 forward, 5'-TCAGATCTCTTCAAGGCTG TGCTG-3', ATP6V0d2 reverse, 5'-GTGCCAAATGAGTTCA GAGTGATG-3'; DC-STAMP forward, 5'-AAAACCCTTGGG CTGTTCTT-3', DC-STAMP reverse, 5'-CTTCGCATGCAG 
GTATTCAA-3'; TRAP forward, 5'-CTGGAGTGCACGATGC CAGCGACA-3', TRAP reverse, 5' ${ }^{\prime}$-TCCGTGCTCGGCGATG GACCAGA-3' ${ }^{\prime}$; CtsK forward, 5'-ACGGAGGCATTGACTCT GAAGATG-3', CtsK reverse, 5'-GTTGTTCTTATTCCGAGC CAAGAG- $3^{\prime}$; c-Src forward, 5'-TTCAACAGCCTGCAGCAG CTCGTG-3', c-Src reverse, 5'-TTCCGACACCACAGCATA CAGCTG- $3^{\prime}$; integrin $\beta 3$ forward, $5^{\prime}$-GATGACATCGAGCA GGTGAAAGAG- $3^{\prime}$, integrin $\beta 3$ reverse, $5^{\prime}$-CCGGTCAT GAATGGTGATGAGTAG- $3^{\prime}$; and GAPDH forward, $5^{\prime}$-AAAC CCATCACCATCTTCCA-3', GAPDH reverse, 5'-GTGGTTCA CACCCATCACAA- $3^{\prime}$. Following cDNA synthesis by reverse transcriptase, PCRs were carried out at $94{ }^{\circ} \mathrm{C}$ for $30 \mathrm{~s}$, $55-60{ }^{\circ} \mathrm{C}$ for $30 \mathrm{~s}$, and $72{ }^{\circ} \mathrm{C}$ for $1 \mathrm{~min}(25-30$ cycles $)$. The PCR products were separated by electrophoresis on $2 \%$ agarose gels and visualized by ethidium bromide staining with UV light illumination.

\section{Western Blotting}

RAW-D cells were starved in $\alpha$-MEM containing $0.5 \%$ FBS for $5 \mathrm{~h}$ followed by incubation with or without NDGA for $60 \mathrm{~min}$. These cells were stimulated with $300 \mathrm{ng} / \mathrm{ml}$ RANKL and further incubated for the indicated times. Thereafter, the cells were collected and lysed in lysis buffer containing $1 \%$ Triton X-100, 0.5\% NP-40, $150 \mathrm{mM}$ $\mathrm{NaCl}, 50 \mathrm{mM}$ Tris-HCl (pH 7.4), $1 \mathrm{mM} \mathrm{NaF}, 1 \mathrm{mM} \mathrm{Na} \mathrm{VO}_{4}$, $1 \mathrm{mM}$ phenylmethylsulfonyl fluoride, and protease inhibitor cocktail (Sigma, St Louis, MO, USA). The lysates containing equal amount of proteins were separated by $10 \%$ SDSPAGE and transferred to nitrocellulose membranes. After blocking with 5\% non-fat dry milk, the membranes were probed with anti-phospho ERK1/2, JNK1/2, or p38 Abs and reacted with peroxidase-conjugated anti-mouse or anti-rabbit IgG Abs. Immunoreactive bands were visualized using an ECL kit (Amersham Biosciences, Buckinghamshire, UK). After stripping bound Abs, the same membranes were reprobed with anti-ERK1/2, JNK1/2, p38, I $\kappa$ B, NFATc1, or actin.

\section{Nuclear Translocation of NFATc1}

Osteoclast-like MNCs from mouse BMM cultures were incubated with RANKL $(50 \mathrm{ng} / \mathrm{ml})$ for $30 \mathrm{~min}$ in the presence or absence of NDGA $(5 \mu \mathrm{M})$, after which the cells were fixed in $4 \% \mathrm{PFA} / \mathrm{PBS}$ ( $10 \mathrm{~min}$, room temperature), washed in PBS, and permeabilized with $0.1 \%$ Triton X-100 ( $5 \mathrm{~min}$, room temperature). After blocking with 5\% goat serum (30 min, room temperature), the cells were incubated with anti-NFATc1 antibody (1:100 in PBS; Santa Cruz Biotechnology) overnight at $4{ }^{\circ} \mathrm{C}$. The cells were washed in PBS and incubated with Cy3-conjugated anti-mouse IgG secondary antibody (1:100 in PBS; Jackson) for $1 \mathrm{~h}$ at room temperature. After rinsing in PBS, the nuclei were stained with DAPI (Invitrogen), and the fluorescent images were examined using fluorescence microscopy (BZ-8100; Keyence).

\section{Intracellular $\mathrm{Ca}^{2+}$ Measurement}

RAW-D cells were cultured with RANKL $(20 \mathrm{ng} / \mathrm{ml})$ for $24 \mathrm{~h}$ in the presence or absence of NDGA $(5 \mu \mathrm{M})$. For intracellular $\mathrm{Ca}^{2+}$ measurement, the cells were incubated with $5 \mu \mathrm{M}$ fluo$4 \mathrm{AM}$ and $0.05 \%$ pluronic F127 for $30 \mathrm{~min}$ in serum-free $\alpha$ MEM, and then washed twice with Hanks' balanced salt solution. The cells were excited at $488 \mathrm{~nm}$, and fluorescence emission at $520 \mathrm{~nm}$ for fluo-4 AM were monitored using an AQUA COSMO $\mathrm{Ca}^{2+}$ imaging system (Hamamatsu Photonics, Hamamatsu, Japan) at $2 \mathrm{~s}$ intervals. To estimate intracellular $\mathrm{Ca}^{2+}$ levels in single cells, the fluorescence intensity of fluo-4 AM from the basal level was calculated and expressed as the percent of maximum ratio increase, which was obtained by the addition of $10 \mu \mathrm{M}$ ionomycin (Sigma) at the termination of experiments.

\section{Luciferase Reporter Assay}

The NF- $\kappa \mathrm{B}$-dependent reporter assay was performed as described previously. ${ }^{26}$ Briefly, RAW-D cells were cotransfected with NF- $\kappa \mathrm{B}$-dependent reporter plasmid p55IgKLuci or control vector p55Luci and the Renilla luciferase plasmid $\mathrm{p} \Delta \mathrm{TK}-\mathrm{RL}$ using a transfection reagent, fugene-6 (Roche Diagnostics, Basel, Switzerland). Cells were cultured for $24 \mathrm{~h}$, and then stimulated with RANKL (100 ng/ $\mathrm{ml}$ ) and various concentrations of NDGA for $24 \mathrm{~h}$. Cells were then collected and luciferase activity was measured using a dual-luciferase reporter assay system (Promega, Madison, WI, USA) on a luminometer (LUMAT LB9507; Berthold Technologies, Bad Wildbad, Germany). Luciferase activity was normalized to the Renilla luciferase activity of a cotransfected $\mathrm{p} \Delta \mathrm{TK}-\mathrm{RL}$ vector to correct for variation in transfection efficiency.

\section{Induction of Adjuvant Arthritis and $\mu \mathrm{CT}$ Analysis}

Adjuvant arthritis was induced as described previously. ${ }^{27}$ Briefly, 5-week-old female Lewis rats (Kyudo, Tosu, Japan) were intradermally injected at the base of the tail with complete Freund's adjuvant (CFA) consisting of $25 \mathrm{mg} / \mathrm{kg}$ heat-killed Mycobacterium butyricum suspended in mineral oil (CFA rats, $n=7$ ). Rats injected with mineral oil alone were used as control, termed 'none' rats $(n=6)$. NDGA $(30 \mathrm{mg} / \mathrm{kg})$ was administrated intraperitoneally every 2 days beginning on day 11 after the adjuvant injection (CFA/ NDGA rats, $n=7$ ), when clinical symptoms (redness or swelling) were detected in the ankle joints. The paw swelling and clinical score were monitored every 2 days. Paw swelling was measured with a digital micrometer (Mitutoyo, Iishikawa, Japan). Clinical scores were determined using a visual scoring method, ${ }^{28}$ which was defined as follows: grade 0 , no swelling; grade 1, detectable swelling in a single digit; grade 2, swelling in more than one digit; grade 3, swelling of all digits and the instep; and grade 4, severe swelling of the paw and ankle. Each limb was graded, yielding a maximum possible score of 16 per animal. The animals were killed at the end of the experiment (day 28), and the hind paws 
(tarsal bone and tibia) were collected and performed bone morphological analysis using a $\mu$ CT Skyscan 1076 scanner (Skyscan, Konitich, Belgium). Scanning was set to $80 \mathrm{kV}$ and $200 \mu \mathrm{A}$, and $9 \mu \mathrm{m}$ of one scan image. We generated a threedimensional reconstruction with InstaRecon/NRecon software (Skyscan) from the data set of scans. We also performed the quantitative analysis of the scanning images at a distance of $2 \mathrm{~mm}$ from the distal growth plate of tibia. The bone volume/total tissue volume (BV/TV), bone surface/ tissue surface (BS/TS), trabecular number (Tb.N), and trabecular separation (Tb.Sp) were calculated.

\section{Histological Analysis}

After $\mu \mathrm{CT}$ scanning, the hind paws of rats were fixed in $4 \%$ paraformaldehyde/PBS, decalcified in 10\% EDTA for 3 weeks at $4{ }^{\circ} \mathrm{C}$, and embedded in paraffin. Sections $(8 \mu \mathrm{m})$ of ankle joints were prepared and stained with hematoxylin/eosin. The sections were also stained for TRAP.

\section{Ethics Committee Approval}

All animal experiments were performed according to the guidelines for 'Care and Use of Animals of Kyushu University'.

\section{RESULTS}

\section{NDGA Inhibits Osteoclast Differentiation In Vitro}

We examined the effects of NDGA on osteoclastogenesis in two in vitro culture systems, (1) the osteoclast precursor cell line RAW-D cells and (2) primary BMMs. NDGA markedly inhibited the formation of TRAP-positive MNCs in RAW-D cell cultures in a dose-dependent manner (Figure 1a). NDGA also significantly inhibited the formation of osteoclasts generated from mouse BMMs (Figure 1b). Incubation of RAW-D cells or BMMs with NDGA $(1-10 \mu \mathrm{M})$ for $72 \mathrm{~h}$ did not produce evidence of cytotoxicity or reduced cell proliferation in the presence or absence of RANKL, indicating that the inhibitory effect of NDGA on osteoclastogenesis was not due to cytotoxicity or reduced cell growth (Figure 1c). In rat bone marrow culture system, NDGA also inhibited formation of osteoclast-like MNCs, which were positive for TRAP and Kat1 antigen, the osteoclast-specific cell-surface antigen (Figure 1d). Treatment of these cultures with NDGA also inhibited the expression of genes associated with osteoclast differentiation, such as ATP6V0d2, DC-STAMP, TRAP, cathepsin K (Ctsk), c-Src, and integrin $\beta 3$ (Figure 1e).

\section{Inhibition of Bone Resorption Activity by NDGA}

Differentiated MNCs exhibit a morphological and functional polarization and begin to resorb mineralized bone surface. To examine the effect of NDGA on the function of mature osteoclasts, we assessed the ability of mature osteoclast-like MNCs formed in the rat whole bone marrow culture system to form pits on dentin slices in the presence or absence of NDGA. NDGA efficiently reduced both the total area of resorption pits and the total number of pits (Figure 2a and b). The inhibitory effect of NDGA on bone resorption activity was also confirmed using BioCoat Osteologic slides (Figure 2c-e).

\section{Suppression of Osteoclast Formation by NGDA Does not Depend on Lipoxygenase Inhibition}

NDGA is a known inhibitor of 5-LO. 5-LO catalyzes the synthesis of 5(S)-hydroxy-6-trans-8,11,14-cis-eicosatetraenoic acid (5(S)-HETE) and leukotriene LTA4 from arachidonic acid. ${ }^{29}$ LTA4 can be further metabolized to the LTB4 or cysteinyl leukotrienes (LTC4, LTD4, and LTE4). ${ }^{29}$ To test whether the inhibitory effects of NDGA on osteoclast formation is dependent on the inhibition of 5-LO activity, 5-LO metabolites, such as 5-HETE, LTB4, and LTC4, were added to RAW-D cell cultures after pretreatment with NDGA. The addition of 5-LO metabolites did not reverse the inhibitory effect of NDGA on osteoclast formation (Figure $3 \mathrm{a}-\mathrm{c}$ ). We also examined the effect of another 5-LO inhibitor, REV-5901, ${ }^{30}$ on osteoclast formation. We found that REV-5901 did not affect osteoclast formation even at the high concentration of $20 \mu \mathrm{M}$ (the $\mathrm{IC}_{50}$ of REV-5901 for 5-LO is $6 \mu \mathrm{M}$; Figure $3 \mathrm{~d}$, left panel). Because NDGA also inhibits 12-LO, we also tested the effect of the 12-LO inhibitor, baicalein, ${ }^{31}$ and found that baicalein $(1-20 \mu \mathrm{M})\left(\mathrm{IC}_{50}\right.$ for 12-LO is $0.12 \mu \mathrm{M}$ ) had no effect on osteoclast formation (Figure $3 \mathrm{~d}$, right panel). Taken together, these results indicate that NDGA suppresses osteoclastogenesis through a mechanism that is independent of the inhibition of lipoxygenase activity.

\section{NDGA Inhibits Nuclear Translocation and Calcium Signaling Induced by RANKL}

It is believed that NFATc1 is a master transcription factor for osteoclast differentiation and regulates the expression of genes associated with osteoclastogenesis. ${ }^{32}$ Therefore, we examined the effects of NDGA on NFATc1 expression. In the RAW-D cell culture system for osteoclastogenesis, the expression of NFATcl mRNA induced by RANKL was markedly inhibited by NDGA treatment in a dosedependent manner (Figure 4a). Addition of NDGA significantly suppressed the level of NFATc1 protein expression induced by RANKL (Figure 4b). The mRNA induction of NFATc1 by RANKL is regulated by an autoamplification mechanism of NFATc1. ${ }^{33}$ We next examined the effect of NDGA on the nuclear localization in osteoclasts. Mature osteoclasts formed from mouse BMMs were incubated with RANKL for $30 \mathrm{~min}$ in the presence or absence of NDGA, and the localization of NFATc1 was examined by immunofluorescence staining. RANKL-treated cells revealed obvious nuclear translocation of NFATc1 (Figure 4c, left panels). However, NDGA treatment significantly suppressed the RANKL-stimulated translocation of NFATc1 to the nuclei (Figure 4c, right panels). These observations suggest that the inhibition of NFATc1 protein expression and blockage of 
a

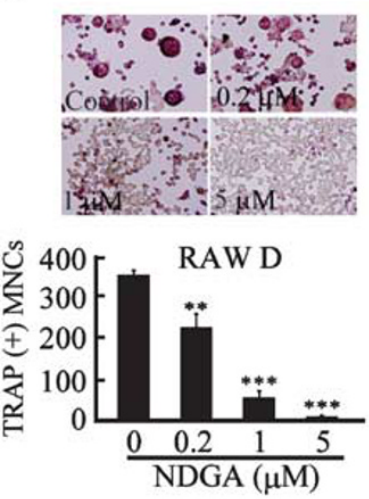

b
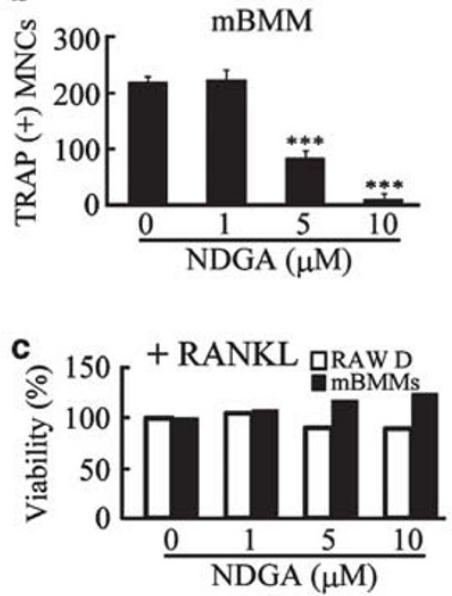

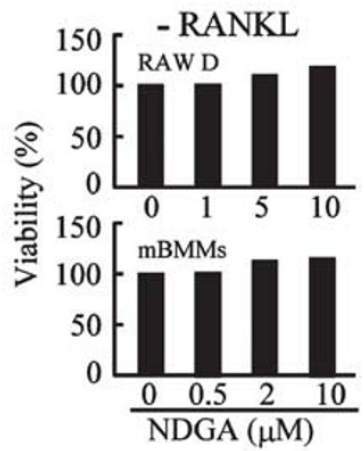

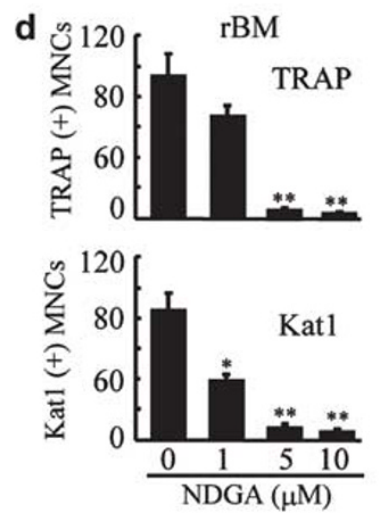

e

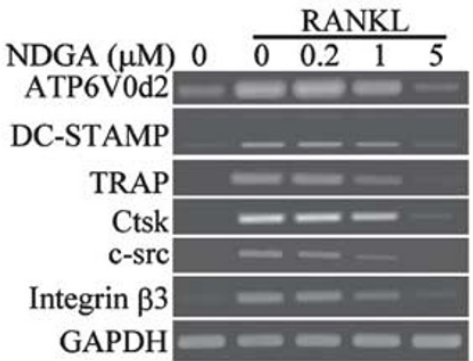

Figure 1 The inhibitory effect of NDGA on osteoclast differentiation in vitro. (a) Dose dependency of NDGA on osteoclastogenesis in RAW-D cell culture (RAW-D). RAW-D cells were cultured for 3 days for forming osteoclast-like MNCs in the presence or absence of the indicated concentrations of NDGA. Upper panel, demonstration of cultures. Original magnification $\times 75$. (b) Dose dependency of NDGA on osteoclastogenesis in BMM culture. Mouse BMMs (mBMMs) were cultured for 5 days for forming osteoclast-like MNCs. Indicated concentrations of NDGA were added to the culture. (c) MTT assay. The left panel: RAW-D cells or mBMMs were cultured for forming osteoclasts, as described above, in the presence or absence of the indicated concentrations of NDGA (+RANKL). MTT assay was performed at 3 days in RAW-D cells and at 5 days in mBMMs, respectively. The right panels: MTT assay was performed also in the absence of RANKL (-RANKL). (d) Suppression of osteoclastogenesis by NDGA in rat bone marrow (rBM) culture system. The rBM cells were cultured for forming osteoclasts as described in Materials and Methods for 4 days followed by staining for TRAP and by osteoclast-specific mAb Kat1. In $\mathbf{a}, \mathbf{b}$, and $\mathbf{d}$, after staining for TRAP, number of TRAP-positive MNCs was counted. In d, number of Kat1-positive MNCs was also counted. Data represent means \pm s.d. $(n=4)$. Data were analyzed by Student's $t$-test. ${ }^{* *} P<0.01$ and ${ }^{* * *} P<0.001$ compared with cultures in the absence of NDGA. (e) Effect of NDGA on gene expression associated with osteoclastogenesis. RAW-D cells were cultured for forming osteoclasts, as described above, in the presence or absence of the indicated concentrations of NDGA. Total RNA was extracted and the mRNA expression levels of the indicated genes were estimated by RT-PCR.

nuclear translocation of NFATc1 are critical steps in the inhibition of osteoclastogenesis by NDGA.

It has been shown that RANKL-induced $\mathrm{Ca}^{2+}$ oscillation is essential for the translocation of NFATc1, which is critical for the sustained NFATc1 nuclear translocation. ${ }^{33}$ We examined the effect of NDGA on $\mathrm{Ca}^{2+}$ oscillation in RAW-D cells stimulated with RANKL. As shown in Figure 4d, RANKL-induced $\mathrm{Ca}^{2+}$ oscillation (upper panel) was markedly inhibited by NDGA (lower panel). These data strongly indicate that the inhibitory effect of NDGA on NFATc1 expression was mediated through inhibition of RANKLinduced $\mathrm{Ca}^{2+}$ oscillation.

\section{Effect of NDGA on RANK Signaling Pathway}

The MAPK and NF- $\kappa$ B signaling pathways downstream of RANK signaling are involved in osteoclastogenesis. We investigated how NDGA affects these signaling pathways. RAWD cells (Figure 5a: the left and right panels) or mouse BMMs (Figure $5 b$ ) were stimulated with RANKL in the presence or absence of NDGA for 0-30 min. RANKL induced rapid phosphorylation of ERK, P38, and JNK (Figure 5a and b, lanes 2-4). The phosphorylation of p38 and JNK was not significantly affected by the addition of NDGA (Figure $5 \mathrm{a}$ and $b$, lanes 6-8). However, NDGA markedly inhibited the phosphorylation of ERK (Figure 5a and b, lanes 6-8). NDGA 

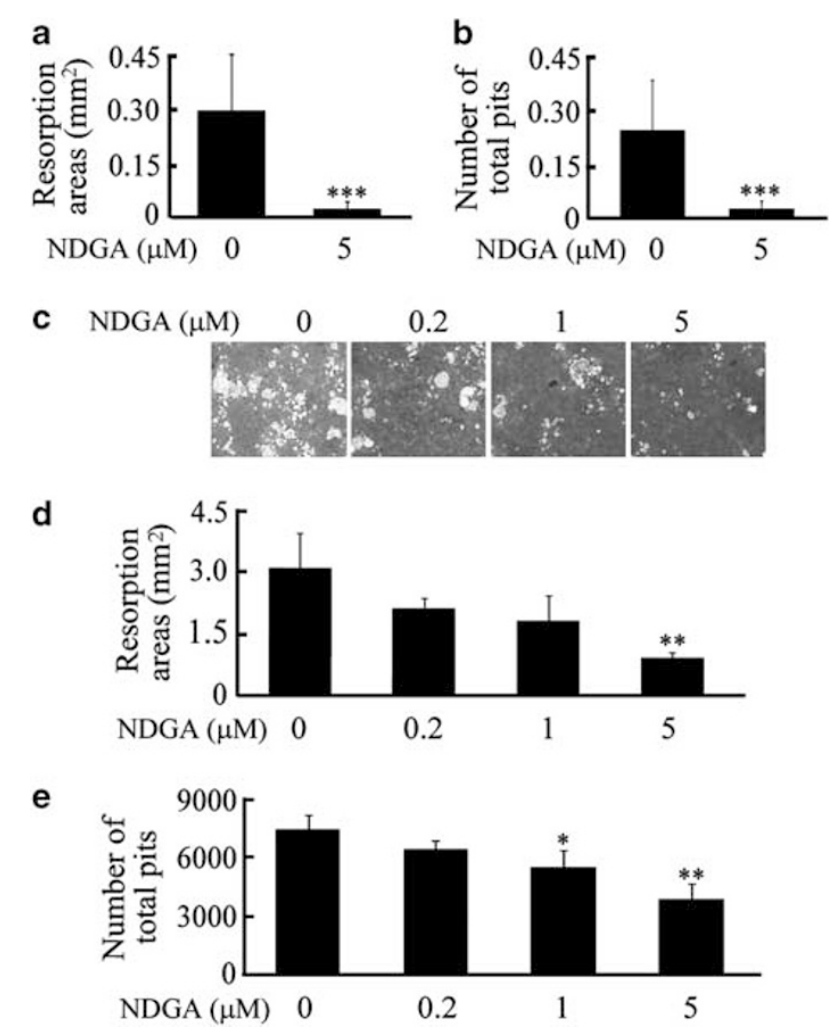

Figure 2 Inhibition of bone resorption by NDGA. Cultures of mature osteoclast-like MNCs obtained from rat whole bone marrow cultures were replated on dentine slices $(\mathbf{a}, \mathbf{b})$ or osteologic slides $(\mathbf{c}-\mathbf{e})$ and cultured for $48 \mathrm{~h}$ in the presence of the indicated concentrations of NDGA. The image of pits (c), total areas of resorption pits $(\mathbf{a}, \mathbf{d})$, and the total number of resorption pits (b, e) were obtained as described in Materials and Methods. Data represent means \pm s.d. $(n=4)$. Data were analyzed by Student's $t$-test. ${ }^{*} P<0.05,{ }^{* *} P<0.01$, and ${ }^{* * *} P<0.001$ compared with the culture without NDGA.

slightly suppressed RANKL-induced $\mathrm{I} \kappa \mathrm{B}$ phosphorylation but not $\mathrm{I} \kappa \mathrm{B}$ degradation (Figure $5 \mathrm{c}$ ). We further evaluated potential effects of NDGA on transcriptional activity of NF$\kappa \mathrm{B}$. RAW-D cells were transiently transfected with a NF- $\kappa \mathrm{B}-$ dependent reporter plasmid p55IgKLuci and stimulated with RANKL in the presence of NDGA. Treatment of NDGA did not significantly affect transcription of the NF- $\kappa \mathrm{B}$ reporter genes (Figure 5d).

\section{Systemic Treatment of NDGA Does not Prevent Development of Arthritis, but Significantly Suppresses Bone Destruction in AA Rats}

To investigate the effects of NDGA in vivo, we analyzed whether NDGA affects bone destruction in AA rats. NDGA $(30 \mathrm{mg} / \mathrm{kg})$ injections were initiated on day 11 after CFA immunization, at which point the slight swelling of joints could be observed. Treatment with NDGA did not significantly decrease joint inflammation, as measured by clinical score (Figure 6a) or amount of swelling of hind paws (Figure 6b). At the end of the experiments, we performed the bone morphological analysis of hind paws using $\mu \mathrm{CT}$. The

three-dimensional bone surface images demonstrating the CFA-injected rats show severe bone destruction in ankle joints (Figure $6 \mathrm{c}, \mu \mathrm{CT}$, middle panel: $\mathrm{CFA}$ ); in contrast, in CFA-injected rats treated with NDGA, bone destruction was significantly suppressed (Figure $6 \mathrm{c}, \mu \mathrm{CT}$, right panel: CFA/ NDGA and Figure 6d), especially in distal tibia. The histological analysis has also shown that the bone destruction observed in distal tibia of AA rats was significantly suppressed by NDGA treatment (Figure 6c, H/E). In addition, numerous TRAP-positive osteoclasts were observed in distal tibia of AA rats (Figure 6c, TRAP, middle panel: CFA and Figure 6e), whereas the number of TRAP-positive osteoclasts in the corresponding area was significantly reduced in NDGA-treated rats (Figure 6c, TRAP, right panel: CFA/ NDGA and Figure 6e). Quantitative analysis of the $\mu \mathrm{CT}$ scanning images at a distance of $2 \mathrm{~mm}$ from the distal growth plate of tibia was performed. The BV/TV, BS/TS, and Tb.N were significantly decreased and the Tb.Sp was clearly increased in AA rats, whereas the treatment of NDGA significantly recovered these parameters (Figure 6d). These 

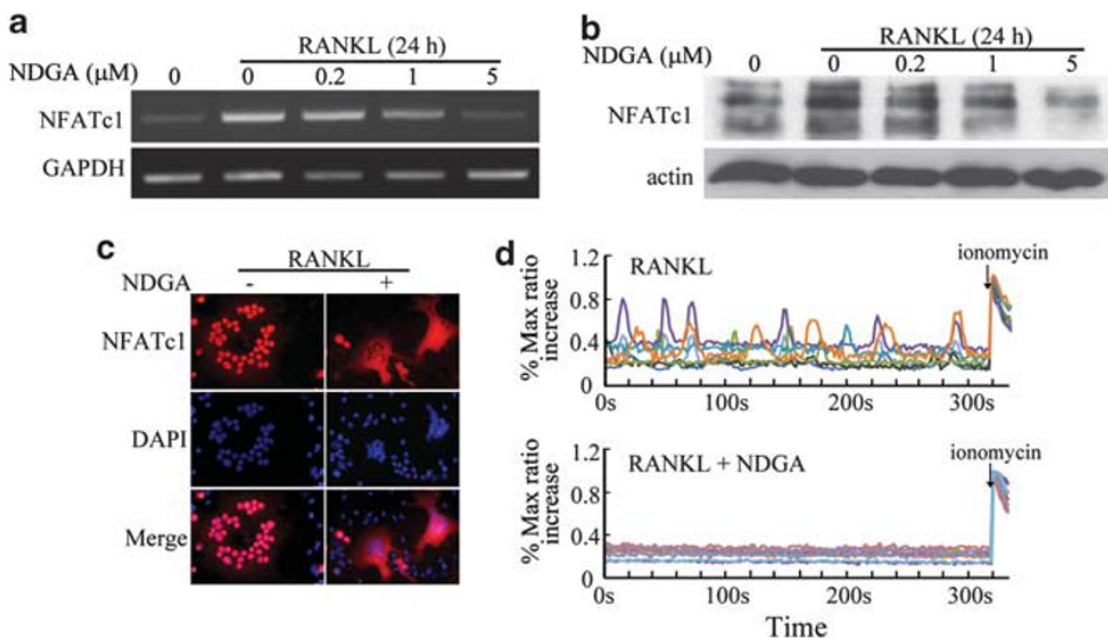

Figure 4 Suppression of RANKL-mediated induction of NFATc1 expression and $\mathrm{Ca}^{2+}$ signaling by NDGA. (a) Semiquantitative RT-PCR to detect NFATc1 mRNA in RANKL-stimulated RAW-D cells. (b) Expression of NFATc1 protein in RAW-D cells. Total cell lysates were prepared and subjected to western blot analysis with anti-NFATc1 and actin Abs. (c) RANKL-induced nuclear translocation of NFATc1 was suppressed by NDGA treatment. Mature osteoclasts from mBMMs were incubated with RANKL $(50 \mathrm{ng} / \mathrm{ml})$ in the absence or presence of NDGA $(5 \mu M)$ for 30 min. The cells were fixed and stained for NFATc1 (red) and nuclei (blue). Original magnification $\times 40$. (d) RANKL-induced $\mathrm{Ca}^{2+}$ oscillation was completely inhibited by NDGA treatment. RAW-D cells were cultured with RANKL $(20 \mathrm{ng} / \mathrm{ml})$ and TNF- $\alpha(1 \mathrm{ng} / \mathrm{ml})$ for $24 \mathrm{~h}$ in the absence (upper panel) or presence (lower panel) of NDGA $(5 \mu \mathrm{M}) .\left[\mathrm{Ca}^{2+}\right] \mathrm{i}$ change in single cells were detected by loading $\mathrm{Ca}^{2+}$ indicators as described in Materials and Methods. Each color indicates corresponding single cells in the same field.

results strongly indicate that NDGA suppresses bone destruction accompanying arthritis through inhibiting osteoclast formation. We further examined the effects of NDGA on normal bone metabolism. Administration of NDGA into normal rats did not show significant effect on bone density, as well as on bone morphology, as shown in Figure $6 f$. These data indicate that NDGA suppressed inflammatory bone destruction without altering the normal bone metabolism.

\section{DISCUSSION}

In this study, we have determined that NDGA effectively inhibited osteoclastogenesis in a direct manner on cells in the osteoclast lineage in murine cell culture systems for evaluating osteoclastogenesis. We also confirmed using rat bone marrow cultures that NDGA significantly suppressed formation of osteoclasts, expressing osteoclast-specific cell surface antigen Kat $1 .{ }^{23-25}$ We also demonstrate the utility of NDGA in the control of inflammatory bone destruction. NFATc1 is a nuclear transcription factor induced by RANKL that is believed to have a central role in RANKL-induced osteoclast differentiation. ${ }^{32}$ In this study, we observed a marked suppression of the levels of NFATc1 mRNA and protein by NDGA treatment. In addition, we found that NDGA markedly inhibited expression of NFATc1-induced genes, ${ }^{32,34-37}$ such as TRAP, Ctsk, integrin $\beta 3$, Atp6v0d2, and DC-STAMP. These results strongly indicate that suppression of NFATc1 induction is a crucial molecular event for the inhibitory effects of NDGA on osteoclast formation. Induction of NFATc1 occurs downstream of RANK signaling following activation of the MAPK and NF- $\kappa$ B pathways. ${ }^{8} 38$ In our study, the activation of p38 and JNK in response to RANKL was not significantly affected by NDGA. NDGA did not affect RANKL-induced I $\kappa$ B phosphorylation and $\mathrm{I} \kappa \mathrm{B}$ degradation involved in NF- $\kappa \mathrm{B}$ signaling, and the transactivity of NF- $\kappa \mathrm{B}$ was not significantly affected by NDGA treatment, suggesting that the NF- $\kappa \mathrm{B}$ pathway does not have an important role in the inhibitory effect of NDGA on NFATc1 induction and osteoclastogenesis. However, NDGA markedly suppressed RANKL-induced activation of ERK. As several reports have demonstrated the essential role of ERK on osteoclast differentiation, ${ }^{39,40}$ inhibitory action of NDGA on ERK activation could result in the suppression of osteoclastogenesis by NDGA.

There have been several studies suggesting that RANKLinduced $\mathrm{Ca}^{2+}$ oscillations are essential for the induction of NFATc1 during osteoclastogenesis. Takayanagi et $a^{32}$ reported that autoamplification of NFATc1 is regulated by calcineurin that was activated by RANKL-induced $\mathrm{Ca}^{2+}$ oscillations during osteoclastogenesis. Koga et $a l^{41}$ as well as Yang and $\mathrm{Li}^{42}$ also reported the important role of $\mathrm{Ca}^{2+}$ oscillation-NFATc1 pathway on osteoclastogenesis. Therefore, we further investigated whether NDGA could affect $\mathrm{Ca}^{2+}$ oscillation induced by RANKL. Interestingly, we could successfully demonstrate a potent inhibitory action of NDGA on RANKL-induced intracellular $\mathrm{Ca}^{2+}$ oscillation in osteoclast precursors. The inhibitory effect of NDGA on intracellular calcium level has been previously described. Culver et $a l^{43}$ reported that NDGA inhibits TNF-induced apoptosis by preventing a rise in the intracellular calcium level in the 3T3-like murine fibroblast cell line. However, the effect of NDGA on $\mathrm{Ca}^{2+}$ oscillation associated with osteoclastogenesis has not been previously reported. In this 
a

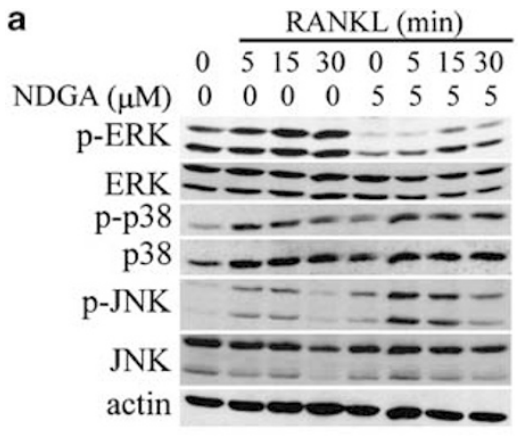

b

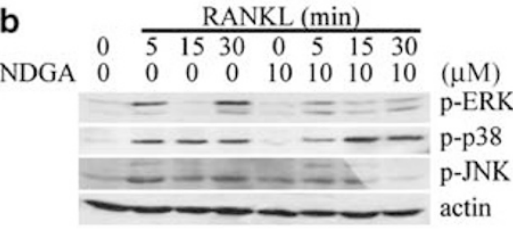

C

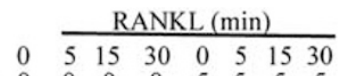

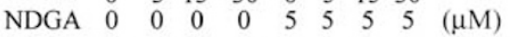

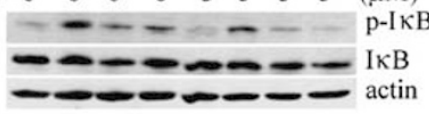

d

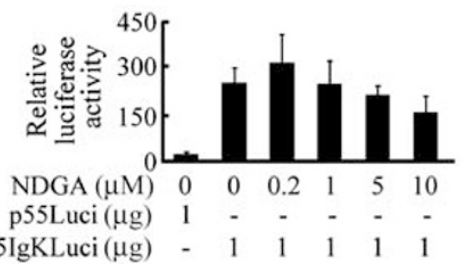

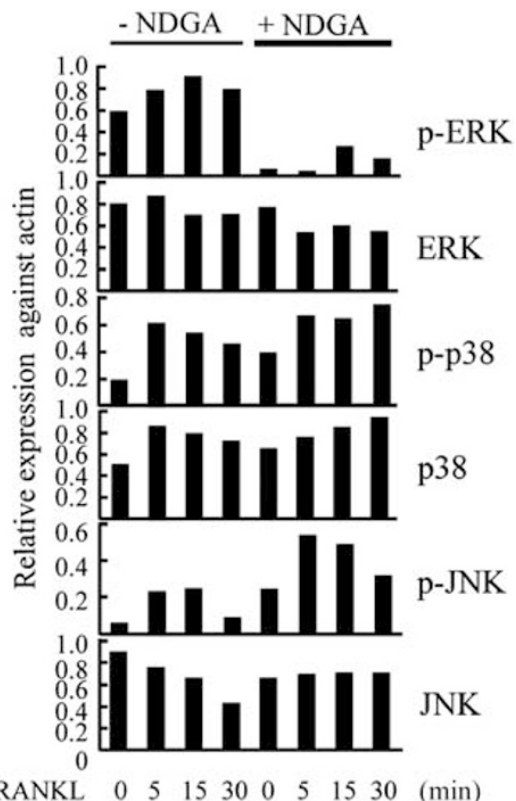

$\begin{array}{llllllllll}\text { RANKL } & 0 & 5 & 15 & 30 & 0 & 5 & 15 & 30 & (\mathrm{~min})\end{array}$

Figure 5 Effects of NDGA on the RANKL signaling pathways. RAW-D cells (a, $\mathbf{c}$ ) or mBMMs (b) were starved in $\alpha$-MEM containing $0.1 \%$ FBS for $5 \mathrm{~h}$ and incubated with or without NDGA for $60 \mathrm{~min}$, followed by stimulation with RANKL $(300 \mathrm{ng} / \mathrm{ml}$ ) for indicated time periods. Total cell lysates were prepared, and equal amount of proteins were subjected to western blot analysis with the indicated Abs. (a, right panels) The density of each band was quantified by using Image J software. The vertical axis demonstrates the ratio of the expression of each protein against that of actin. (d) Luciferase assay was performed to evaluate NF- $\kappa$ B transcription. RAW-D cells were transiently transfected with NF- $\kappa$ B-dependent reporter plasmid p55IgKLuci or control vector p55Luci. Transfected cells were cultured for $24 \mathrm{~h}$ and then stimulated with RANKL ( $100 \mathrm{ng} / \mathrm{ml})$ and varying concentrations of NDGA.

The cells were then collected and the luciferase activity was measured.

study, we have also demonstrated that NDGA markedly suppressed translocation of NFATc1 to the nuclei of mature osteoclasts. Our data indicate that NDGA inhibits induction of NFATc1 through suppressing calcium signaling, which could result in the suppression of osteoclastogenesis. Activation of ERK is also known to associate with calcium signaling. ${ }^{44}$ In neurons, signaling of ERK is required for the activation of nuclear substrates during induction of longterm potentiation, a persistent increase in synaptic strength following high-frequency stimulation of chemical synapse, which is considered a cellular correlate of learning and memory. ${ }^{44}$ Activation of ERK propagates signals generated by synaptically evoked calcium into the cell nucleus. In osteoclast precursors, there could be some relationship between the alteration in the level of ERK phosphorylation and the modulation of $\mathrm{Ca}^{2+}$ oscillation. Further studies are required to determine the detailed molecular events concerning the suppression of RANKL-induced $\mathrm{Ca}^{2+}$ oscillation and osteoclastogenesis by NDGA. NDGA significantly inhibited phosphorylation of ERK without affecting the expression of ERK protein. NDGA could directly bind to ERK protein itself to induce some conformational change, which affects phosphorylation. It is also possible that NDGA inhibits MAPK upstream of ERK1/2, which results in the suppression of ERK phosphorylation. More direct evidence concerning the target molecule of NDGA is required to address the mechanism of ERK inhibition by NDGA.

Although the biological activities of NDGA are generally dependent on its anti-lipoxygenase activity, biological functions independent from this mechanism have also been reported. ${ }^{43,45}$ NDGA regulates IFN- $\gamma$-mediated inflammation through mechanisms unrelated to the inhibition of 5-LO. ${ }^{43}$ In this work, we investigated the inhibitory mechanism of NDGA on osteoclastogenesis. Rev-5901, ${ }^{30}$ another 5-LO inhibitor, showed no apparent inhibitory effect on osteoclast 
a
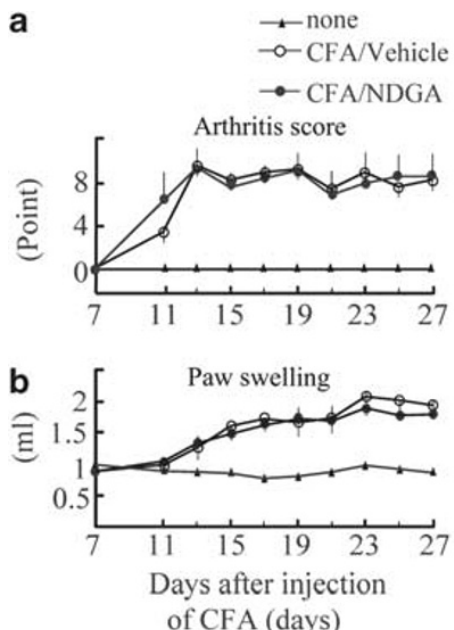

C

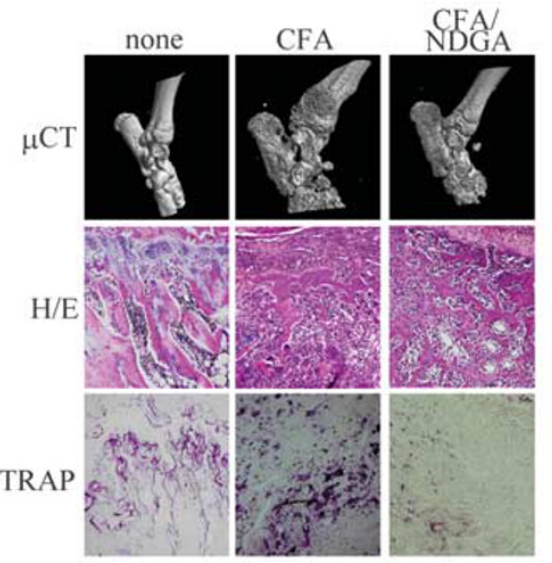

d
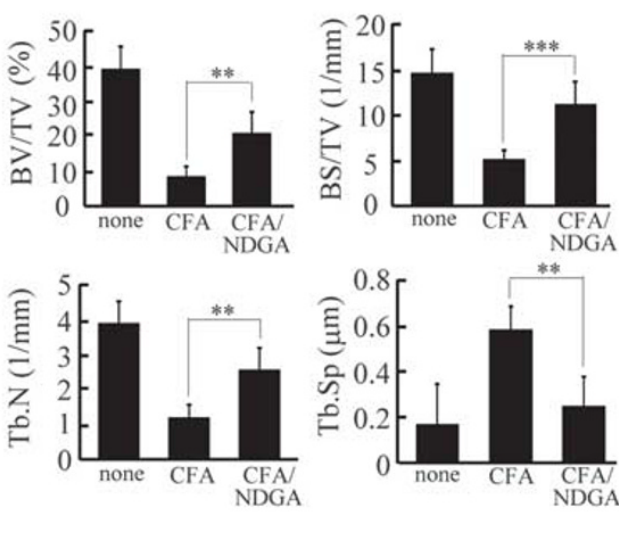

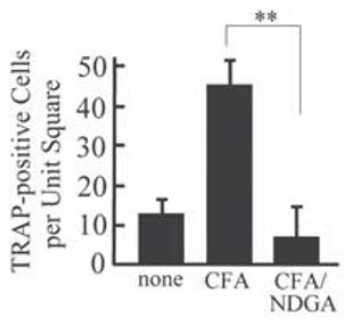

f

none

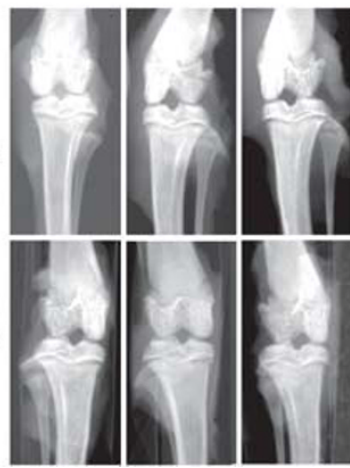

Figure 6 Significant suppression of inflammatory bone destruction by NDGA in AA rats. NDGA $(30 \mathrm{mg} / \mathrm{kg})$ was injected i.p. into CFA-injected rats on day 11. Injections were given once every 2 days until the end of experiments (day 28). The clinical score (a) and swelling of hind paws (b) were monitored on the indicated days. (c) AA rats were treated with NDGA as described above. At 28 days after CFA injection, hind paws were collected and analyzed by $\mu \mathrm{CT}$. Upper panels show the three-dimensional surface images of bones in the ankle joints. Distal parts of the tibia were analyzed histologically by H\&E staining (middle panels) and TRAP staining (lower panels). (d) Data showing the quantitative analysis of the $\mu C T$ scanning images at a distance of $2 \mathrm{~mm}$ from the distal growth plate of tibia. BV/TV, bone volume per tissue volume; BS/TS, bone surface per tissue surface; Tb.N, trabecular number; Tb.Sp, trabecular separation. ${ }^{* *} P<0.01,{ }^{* * *} P<0.01(n=7)$. (e) Quantitative demonstration of osteoclastogenesis in AA rats treated with or without NDGA. Number of TRAP-positive cells was counted per unit area $\left(500 \mu \mathrm{m}^{2}\right)$ of the TRAP-stained sections of the distal tibia $(6$ unit-areas from three independent sections). ${ }^{* * *} P<0.01(n=6)$. (f) Effect of NDGA on normal bone metabolism. NDGA was administered intraperitoneally to normal Lewis rats $(30 \mathrm{mg} / \mathrm{kg}$ ) every 2 days for 26 days followed by the $\mu \mathrm{CT}$ analysis. Radiographs of sagittal axis for control rats ('none'), to which vehicle PBS was injected, and NDGA-treated rats ('NDGA') are demonstrated. Data show radiographs of tibia and femur involving knee joints from three different rats for each experimental group.

formation in RAW-D cell cultures. Furthermore, NDGAinduced inhibition of osteoclastogenesis was not reversed by the addition of 5-LO metabolites. NDGA is also known to inhibit 12-LO; however, a 12-LO-specific inhibitor baicalein $^{31}$ showed no effect on osteoclast formation in our experiments. These results indicate that the mechanism by which NDGA suppresses osteoclastogenesis is not through inhibiting 5-LO and/or 12-LO enzyme activity.

Micro CT and histological analyses of AA rats treated with or without NDGA clearly demonstrated that the bone destruction of ankle joints, including distal tibia and tarsal bone, was significantly prevented by NDGA. In addition, the marked reduction of osteoclastogenesis was apparent in NDGA-treated AA rats. Anti-osteoclastogenic reagents, such as OPG and bisphosphonates, have successfully prevented bone destruction in animal models of arthritis. ${ }^{11-14}$ The inhibitory effect of NDGA on bone destruction in AA rats is thought to be mediated through the direct action of NDGA on osteoclastogenesis at the site of bone destruction. This hypothesis is also supported by our in vitro experiments, in which NDGA markedly inhibited osteoclast formation through suppressing NFATcl signaling as well as blocking RANKL-induced $\mathrm{Ca}^{2+}$ oscillation. Thus, NDGA treatment should provide another ideal medication for controlling inflammatory bone destruction. Administration of NDGA did not affect normal bone metabolism in normal rats, however, NDGA slightly suppressed formation of osteoblastlike cell colonies from bone marrow cells (data not shown). Further studies are required to conclude the effects of NDGA on osteoblastogenesis. 
Several reports have suggested that NDGA possesses antiinflammatory activity. ${ }^{17,46}$ Culver et $a l^{43}$ and Eads et al ${ }^{47}$ reported that NDGA and its methylated derivative, terameprocol, inhibit production of TNF- $\alpha$ and several other inflammatory cytokines induced by LPS. However, in this study, inflammatory parameters, such as paw swelling, and inflammatory scores in AA rats were not significantly reduced by NDGA treatment. To investigate the direct effect of NDGA on osteoclastogenesis in vivo, we have performed NDGA treatment after the onset of inflammation in AA rats (on day 11). Although in this study the inflammation of AA was not significantly inhibited by NDGA treatment, our data do not exclude a possibility that earlier administration of NDGA may inhibit the inflammation of arthritis. Nakamura et $a l^{48}$ reported that the therapeutic treatment (on day 11) with FR901228T does not suppress paw swelling but markedly suppresses bone destruction in AA rats. ${ }^{45}$ In contrast, the prophylactic treatment of AA rats (on day 0) with FR901228T completely suppresses paw swelling as well as bone destruction. Chung et $a l^{49}$ also reported similar results. They found that early treatment of AA with TSA, before the onset of AA, inhibited the inflammation, but that late therapeutic treatment had no significant effect. Prophylactic effect of NDGA on bone destruction would also be addressed in future studies.

In conclusion, we found that the NDGA markedly suppressed osteoclast differentiation through suppressing the induction of NFATc1 possibly via inhibition of calcium oscillation. This is the first report to demonstrate an inhibitory function of NDGA on osteoclastogenesis. We also demonstrated a potent inhibitory function of NDGA on bone destruction in AA rats. Our present study could lead to the development of NDGA as an effective medicine to prevent pathological bone resorption observed in various bone diseases, such as RA. Preceding clinical researches to develop NDGA as a novel anticancer medicine would facilitate the application of this compound to develop as the effective medicine for inflammatory bone destruction.

\section{ACKNOWLEDGEMENTS}

We thank Dr Eric Milner for English language editing. This work was funded in part by a Grant-in-Aid for Scientific Research of Japan, Grant numbers 21390492, 21592332, 22659330, and 21659424.

\section{DISCLOSURE/CONFLICT OF INTEREST}

The authors declare no conflict of interest.

1. Teitelbaum SL. Bone resorption by osteoclasts. Science 2000;289: 1504-1508.

2. Boyle WJ, Simonet WS, Lacey DL. Osteoclast differentiation and activation. Nature 2003;423:337-342.

3. Gravallese EM, Harada Y, Wang JT, et al. Identification of cell types responsible for bone resorption in rheumatoid arthritis and juvenile rheumatoid arthritis. Am J Pathol 1998;152:943-951.

4. Kong YY, Feige U, Sarosi I, et al. Activated T cells regulate bone loss and joint destruction in adjuvant arthritis through osteoprotegerin ligand. Nature 1999;402:304-309.
5. Li YJ, Kukita A, Teramachi J, et al. A possible suppressive role of galectin-3 in upregulated osteoclastogenesis accompanying adjuvantinduced arthritis in rats. Lab Invest 2009;89:26-37.

6. Toh K, Kukita T, Wu Z, et al. Possible involvement of MIP-1alpha in the recruitment of osteoclast progenitors to the distal tibia in rats with adjuvant-induced arthritis. Lab Invest 2004;84:1092-1102.

7. Suda $\mathrm{T}$, Takahashi N, Udagawa $\mathrm{N}$, et al. Modulation of osteoclast differentiation and function by the new members of the tumor necrosis factor receptor and ligand families. Endocr Rev 1999;20: 345-357.

8. Takayanagi $\mathrm{H}$. The role of NFAT in osteoclast formation. Ann N Y Acad Sci 2007;1116:227-237.

9. Takayanagi $\mathrm{H}$, lizuka $\mathrm{H}$, Juji $\mathrm{T}$, et al. Involvement of receptor activator of nuclear factor kappaB ligand/osteoclast differentiation factor in osteoclastogenesis from synoviocytes in rheumatoid arthritis. Arthritis Rheum 2000;43:259-269.

10. Simonet WS, Lacey $\mathrm{DL}$, Dunstan $\mathrm{CR}$, et al. Osteoprotegerin: a novel secreted protein involved in the regulation of bone density. Cell 1997;89:309-319.

11. Romas E, Sims NA, Hards DK, et al. Osteoprotegerin reduces osteoclast numbers and prevents bone erosion in collagen-induced arthritis. Am J Pathol 2002:161:1419-1427.

12. Harada $\mathrm{H}$, Nakayama $\mathrm{T}$, Nanaka $\mathrm{T}$, et al. Effects of bisphosphonates on joint damage and bone loss in rat adjuvant-induced arthritis. Inflamm Res 2004;53:45-52.

13. Itoh $\mathrm{F}$, Aoyagi $\mathrm{S}$, Kusama $\mathrm{H}$, et al. Effects of clodronate and alendronate on local and systemic changes in bone metabolism in rats with adjuvant arthritis. Inflammation 2004;28:15-21.

14. Herrak P, Gortz B, Hayer S, et al. Zoledronic acid protects against local and systemic bone loss in tumor necrosis factor-mediated arthritis. Arthritis Rheum 2004;50:2327-2337.

15. Pettit $A R$, Ji $H$, von Stechow $D$, et al. TRANCE/RANKL knockout mice are protected from bone erosion in a serum transfer model of arthritis. Am J Pathol 2001;159:1689-1699.

16. Redlich K, Hayer S, Ricci R, et al. Osteoclasts are essential for TNFalpha-mediated joint destruction. J Clin Invest 2002;110:1419-1427.

17. Arteaga S, Andrade-Cetto A, Cardenas R. Larrea tridentata (Creosote bush), an abundant plant of Mexican and US-American deserts and its metabolite nordihydroguaiaretic acid. J Ethnopharmacol 2005; 98:231-239.

18. Youngren JF, Gable K, Penaranda C, et al. Nordihydroguaiaretic acid (NDGA) inhibits the IGF-1 and c-erbB2/HER2/neu receptors and suppresses growth in breast cancer cells. Breast Cancer Res Treat 2005; 94:37-46.

19. Ryan CJ, Zavodovskaya M, Youngren JF, et al. Inhibitory effects of nordihydroguaiaretic acid (NDGA) on the IGF-1 receptor and androgen dependent growth of LAPC-4 prostate cancer cells. Prostate 2008;68:1232-1240.

20. Watanabe T, Kukita T, Kukita A, et al. Direct stimulation of osteoclastogenesis by MIP-1alpha: evidence obtained from studies using RAW264 cell clone highly responsive to RANKL. J Endocrinol 2004;180:193-201.

21. Kukita A, Kukita $\mathrm{T}$, Shin $\mathrm{JH}$, et al. Induction of mononuclear precursor cells with osteoclastic phenotypes in a rat bone marrow culture system depleted of stromal cells. Biochem Biophys Res Commun 1993;196:1383-1389.

22. Kukita A, Kukita T, Hata K, et al. Heat-treated osteoblastic cell (ROS17/ 2.8)-conditioned medium induces the formation of osteoclast-like cells. Bone Miner 1993;23:113-127.

23. Kukita T, Kukita A, Nagata K, et al. Novel cell-surface Ag expressed on rat osteoclasts regulating the function of the calcitonin receptor. J Immunol 1994;153:5265-5273.

24. Kukita T, Kukita A, Xu L-X, et al. Successful detection of active osteoclasts in situ by systemic administration of an osteoclast-specific monoclonal antibody. Calcif Tissue Int 1998;63:148-153.

25. Kukita T, Kukita A, Xu L-X, et al. Kat1-antigen-a reliable immunological marker for identifying osteoclast precursors of rats: detection of subpopulations among precursors and initiation of osteoclastogenesis. Histochem Cell Biol 2001;115:215-222.

26. Rahman MM, Kukita A, Kukita $\mathrm{T}$, et al. Two histone deacetylase inhibitors, trichostatin A and sodium butyrate, suppress differentiation into osteoclasts but not into macrophages. Blood 2003;101: 3451-3459. 
27. Kuratani T, Nagata K, Kukita T, et al. Induction of abundant osteoclastlike multinucleated giant cells in adjuvant arthritic rats with accompanying disordered high bone turnover. Histol Histopathol 1998;13:751-759.

28. Hossain $A$, Zheng $C L$, Kukita $A$, et al. Balance of Th1/Th2 cytokines associated with the preventive effect of incomplete Freund's adjuvant on the development of adjuvant arthritis in LEW rats. J Autoimmun 2001;17:289-295.

29. Duroudier NP, Tulah AS, Sayers I. Leukotriene pathway genetics and pharmacogenetics in allergy. Allergy 2009;64:823-839.

30. Kusner EJ, Marks RL, Aharony D, et al. Inhibition by REV-5901 of leukotriene release from guinea-pig and human lung tissue in vitro. Biochem Pharmacol 1989;38:4183-4189.

31. Lebeau A, Esclaire F, Rostene W, et al. Baicalein protects cortical neurons from beta-amyloid (25-35) induced toxicity. Neuroreport 2001;12:2199-2202.

32. Takayanagi $\mathrm{H}, \mathrm{Kim} \mathrm{S}$, Koga $\mathrm{T}$, et al. Induction and activation of the transcription factor NFATC1 (NFAT2) integrate RANKL signaling in terminal differentiation of osteoclasts. Dev Cell 2002;3:889-901.

33. Asagiri $M$, Sato $K$, Usami $T$, et al. Autoamplification of NFATc1 expression determines its essential role in bone homeostasis. J Exp Med 2005;202:1261-1269.

34. Matsumoto M, Kogawa M, Wada S, et al. Essential role of p38 mitogenactivated protein kinase in cathepsin $\mathrm{K}$ gene expression during osteoclastogenesis through association of NFATc1 and PU.1. J Biol Chem 2004;279:45969-45979.

35. Crotti TN, Flannery $M$, Walsh NC, et al. NFATC1 regulation of the human beta3 integrin promoter in osteoclast differentiation. Gene 2006;372:92-102.

36. Kim K, Lee SH, Ha Kim J, et al. NFATc1 induces osteoclast fusion via upregulation of Atp6v0d2 and the dendritic cell-specific transmembrane protein (DC-STAMP). Mol Endocrinol 2008;22:176-185.

37. Kukita $T$, Wada $N$, Kukita $A$, et al. RANKL-induced DC-STAMP is essential for osteoclastogenesis. J Exp Med 2004;200:941-946.

38. Takatsuna $\mathrm{H}$, Asagiri $\mathrm{M}$, Kubota $\mathrm{T}$, et al. Inhibition of RANKL-induced osteoclastogenesis by (-)-DHMEQ, a novel NF-kappaB inhibitor, through downregulation of NFATc1. J Bone Miner Res 2005;20:653-662.
39. Lee SE, Chung WJ, Kwak HB, et al. Tumor necrosis factor-alpha supports the survival of osteoclasts through the activation of Akt and ERK. J Biol Chem 2001;276:49343-49349.

40. David JP, Sabapathy K, Hoffmann O, et al. JNK1 modulates osteoclastogenesis through both c-Jun phosphorylation-dependent and -independent mechanisms. J Cell Sci 2002;115(Pt 22):4317-4325.

41. Koga $T$, Inui $M$, Inoue $K$, et al. Costimulatory signals mediated by the ITAM motif cooperate with RANKL for bone homeostasis. Nature 2004; 428:758-763.

42. Yang S, Li YP. RGS10-null mutation impairs osteoclast differentiation resulting from the loss of $\left[\mathrm{Ca}^{2+}\right]$ i oscillation regulation. Genes Dev 2007:21:1803-1816.

43. Culver CA, Michalowski SM, Maia RC, et al. The anti-apoptotic effects of nordihydroguaiaretic acid: inhibition of CPLA(2) activation during TNF-induced apoptosis arises from inhibition of calcium signaling. Life Sci 2005;77:2457-2470.

44. Wiegert JS, Bading $\mathrm{H}$. Activity-dependent calcium signaling and ERK-MAP kinases in neurons: a link to structural plasticity of the nucleus and gene transcription regulation. Cell Calcium 2011;49: 296-305.

45. Meyer GE, Chesler L, Liu D, et al. Nordihydroguaiaretic acid inhibits insulin-like growth factor signaling, growth, and survival in human neuroblastoma cells. J Cell Biochem 2007;102:1529-1541.

46. Jeon SB, Ji KA, You HJ, et al. Nordihydroguaiaretic acid inhibits IFNgamma-induced STAT tyrosine phosphorylation in rat brain astrocytes. Biochem Biophys Res Commun 2005;328:595-600.

47. Eads D, Hansen R, Oyegunwa A, et al. Terameprocol, a methylated derivative of nordihydroguaiaretic acid, inhibits production of prostaglandins and several key inflammatory cytokines and chemokines. J Inflamm (Lond) 2009;6:2.

48. Nakamura $\mathrm{T}$, Kukita $\mathrm{T}$, Shobuike $\mathrm{T}$, et al. Inhibition of histone deacetylase suppresses osteoclastogenesis and bone destruction by inducing IFN-beta production. J Immunol 2005;175:5809-5816.

49. Chung YL, Lee MY, Wang AJ, et al. A therapeutic strategy uses histone deacetylase inhibitors to modulate the expression of genes involved in the pathogenesis of rheumatoid arthritis. Mol Ther 2003; 8:707-717 